\title{
Novedades florísticas y corológicas para la provincia de Córdoba (España)
}

Juan Antonio Devesa', Gloria Martínez-Sagarra', Emilio Reyes Sepúlveda² y Javier López-Tirado ${ }^{3}$

'Departamento de Botánica, Ecología y Fisiología Vegetal, Facultad de Ciencias, Universidad de Córdoba. Edificio José Celestino Mutis, Campus de Rabanales, Universidad de Córdoba, 14071 (Córdoba).

${ }^{2} \mathrm{C} /$ María la Judía 10, $2^{\circ}$ 1-D, 14011-Córdoba.

${ }^{3}$ Departamento de Ciencias Integradas, Facultad de Ciencias Experimentales, Campus de El Carmen, Universidad de Huelva, 21071 (Huelva).

\section{Correspondencia}

J.A. Devesa

e-mail: bv1dealj@uco.es

Recibido: 20 julio 2918

Aceptado: 5 octubre 2018

Publicado on-line: octubre 2018

\section{Floristic and chorologic novelties for the Flora of Cordoba province}

Palabras clave: Flora vascular, Corología, Córdoba.

Key words: Vascular flora, Chorology, Córdoba.
Se comentan 13 taxones de plantas vasculares de interés para la flora de Córdoba, ya sea porque se amplía significativamente su conocimiento corológico en la provincia [Isoetes velatum A. Braun subsp. velatum, Marsilea strigosa Willd., Phytolacca americana L., Rumex palustris Sm., Carum verticillatum (L.) W. D. J. Koch, y Holcus mollis L.], se confirma su presencia (Lupinus micranthus Guss.) o bien son novedad para el territorio [Armeria linkiana Nieto Fel., Limonium sinuatum (L.) Mill., Maclura pomifera (Raf.) C. K. Schneid., Schoenoplectus erectus (Poir.) Palla ex J. Raynal, y Cenchrus longisetus M. C. Johnst.]. En el caso de Eryngium caespitiferum Font Quer \& Pau, su nueva recolección respalda la primera cita dada a conocer para Europa. Todos los pliegos-testimonio se encuentran depositados en el Herbario de la Universidad de Córdoba (COFC).

\section{PTERIDOPHYTA}

Isoetes velatum A. Braun in Bory \& Durieu, Expl. Sci. Algérie, Atlas, pl. 37, fig. 1 (1849) subsp. velatum

CÓRDOBA. Laguna de Los Arenales, término municipal de Puente Genil, 30SUG4944, 325 m.s.n.m., 29-VI-2018, J.A. Devesa \& J. LópezTirado (COFC 65786).

Taxón distribuido por el oeste de la Región Mediterránea, que aparece disperso por buena parte de la Península Ibérica (Prada, 1986), siendo más raro en el este (Moreno et al., 2015). En la provincia de Córdoba se conoce solo de la mitad septentrional (Ruiz de Clavijo \& Muñoz, 1985; Jiménez \& Ruiz de Clavijo, 1990; Melendo, 1998; Delgado et al., 2007; Moreno et al., loc. cit.; López Tirado et al., 2016). La población encontrada extiende su área de distribución al Valle del Guadalquivir.

Marsilea strigosa Willd., Sp. PI. 5(1): 539 (1810) CÓRDOBA. Inmediaciones del arroyo Majavacas, término municipal de Fuente Obejuna, 30STH8741, 545 m.s.n.m., 20-V-2018, E. Reyes (COFC 65787).

Especie de la Región Mediterránea y el sur de Rusia (Paiva, 1986), que en la Península Ibérica aparece esporádicamente sobre todo en el centro y el cuadrante suroeste (Moreno et al., 2015). En la provincia de Córdoba [aunque no indicada por Paiva (loc. cit.) ni por Cabezudo \& Salvo (1987)], su presencia ha sido acreditada con posterioridad en la depresión del Guadalquivir (Prunier \& Delgado, 2010; Muñoz et al., 2016). En la mitad norte de la provincia solo se conoce del extremo oriental de la comarca de Los Pedroches (Moreno et al., loc. cit.), por lo que la población que ahora se da a conocer amplía notablemente el área de la especie al extremo noroccidental de la provincia, a casi $100 \mathrm{~km}$ de la población pedrocheña y unos $70 \mathrm{~km}$ respecto de la Charca de Rabanales, en las inmediaciones de la ciudad de Córdoba. Las plantas han sido localizadas en una pequeña charca, seca durante el verano, junto con Eleocharis palustris (L.) Roem. \& Schult., Isoetes velatum A. Braun subsp. velatum, Eryngium galioides Lam., Exaculum 
pusillum (Lam.) Caruel, y Lythrum borysthenicum (Schrank) Litv., entre otras especies. La población se ubica justo en el área en la que $M$. batardae Launert es la especie que predomina en la zona.

\section{DICOTYLEDONEAE}

Phytolacca americana L. Sp. PI.: 441 (1753)

CÓRDOBA. Río Yeguas, en su intersección con la carretera de Azuel a Fuencaliente, 30SUH8446, 528 m.s.n.m., 10-VI-2018, J.A. Devesa (COFC 65782).

Neófito originario del norte de América (Nogueira, 1990) que en la Península Ibérica aparece naturalizado sobre todo en las provincias de la periferia, más rara vez hacia el interior (vide Anthos). Aunque Nogueira (loc. cit.) no señaló su presencia en la provincia de Córdoba, la especie había sido ya dada a conocer para las inmediaciones de Palma del Río y Hornachuelos (Fernández, 1986; Pujadas, 1986; Pastor, 1987), zona en la que es relativamente frecuente formando parte de la vegetación riparia del río Guadalquivir, y en la del tramo bajo del río Bembézar. Su hallazgo en el límite con la provincia de Ciudad Real amplía significativamente su área provincial, y extiende su presencia a la comarca de Pedroches.

Armeria linkiana Nieto Fel. in Anales Jard. Bot. Madrid 44: 332 (1987)

CÓRDOBA. La Salinera, Vereda de Granja de Torrehermosa, término municipal de Fuente Obejuna, 30STH8640, 550 m.s.n.m., 20-V-2018, E. Reyes (COFC 65776). Las Patudas, término municipal de Hinojosa del Duque, 30STH9953, 550 m.s.n.m., 26-IV-2008, E. Reyes (COFC 65777).

Especie endémica de la Península lbérica, cuyo material tipo procede del Algarve (Nieto, 1987). Su área se extiende además por el Baixo Alentejo, en Portugal, y también por las provincias españolas de Huelva y Sevilla (Nieto, 1990). Su hallazgo en el norte de la provincia de Córdoba constituye una novedad provincial, y una ampliación importante de su área de distribución.

Maclura pomifera (Raf.) C. K. Schneid., III. Handb. Laubholzk. 1: 806 (1906)

loxylon pomiferum Raf. in Amer. Monthly Mag. \& Crit. Rev. 2: 118 (1817)

CÓRDOBA. Arroyo de la Parrilla, término municipal de Fuente Obejuna, 30STH9841, 500 m.s.n.m., 8-VII-2018, E. Reyes (COFC 65789).

El naranjo de Luisiana, como se conoce, es un árbol caducifolio originario del norte de América, que se cultiva ocasionalmente para hacer setos o como ornamental, con dura madera y frutos carnosos que recuerdan a las naranjas, aunque son amarillos (López González, 2001). La planta es potencialmente invasora y, de hecho, así se la contempla en el listado de plantas alóctonas invasoras (Sanz et al., 2001), y como tal figura ya en la flora portuguesa (Domingues de Almeida \& Freitas, 2006; Domingues de Almeida, 2012), en la denominada cuenca Internacional del Guadiana (Bejarano et al., 2011), en la flora de Vizcaya (Herrera \& Campos, 2010) y, como potencialmente invasora, en la flora del Principado de Asturias (González, 2007). Se han localizado varios ejemplares naturalizados en las orillas de un arroyo, junto con Populus nigra L., Ulmus minor Mill., Ficus carica L., Nerium oleander L. subsp. oleander, y Rubus ulmifolius Schott.

Rumex palustris Sm., Fl. Brit. 1: 394 (1800)

CÓRDOBA. Presa de Martín Gonzalo, arroyo bajo la presa, término municipal de Montoro, 30SUH8216, 230 m.s.n.m., 10-VI-2018, J.A. Devesa (COFC 65783).

Especie cuya área de distribución se extiende por Europa, el oeste de Asia, y el noroeste de África (López, 1990). Su distribución en la Península Ibérica, y en la provincia de Córdoba en particular, ha sido recientemente comentada (Reyes et al., 2017), apuntando para esta última su presencia en la Campiña y en las Sierras Subbéticas, ambas al sur del río Guadalquivir. La población detectada ahora amplía su presencia a Sierra Morena.

Limonium sinuatum (L.) Mill., Gard. Dict., ed. 8: Limonium no. 6 (1768)

Statice sinuata L., Sp. PI.: 276 (1753)

CÓRDOBA. Paraje de la Almarja, Hornachuelos, 30SUG0188, 151 m.s.n.m., 1-V2018, J.A. Devesa \& G. Martínez-Sagarra (COFC 65784).

Especie propia de marismas y arenales marítimos, cuya área se extiende por la región Mediterránea, si bien aparece naturalizada en algunas regiones de Europa (vide Euro+Med PlantBase). En la Península Ibérica se distribuye por las provincias litorales del sur y este de España (Devesa, 1987; Crespo \& Lledó, 1998; Erben, 2006; Royo, 2006; Serra, 2007, etc.; vide Anthos). Se utiliza a menudo en jardinería (Trigo, 2000), y con toda seguridad este es el origen de la pequeña población localizada en las inmediaciones de Hornachuelos, que integran poco más de una docena de especímenes, plenamente integrados en herbazales nitrófilos, pero cuya supervivencia en el futuro está por ver. 
Lupinus micranthus Guss., Fl. Sicul. Prodr. 2: 400 (1828-1832)

CÓRDOBA. Arroyo Pedroches, término municipal de Córdoba, 30SUG4597, 120 m.s.n.m., 3-VI-2018, E. Reyes (COFC 65788).

Planta de la Región Mediterránea cuya presencia provincial fue dada a conocer en Flora iberica (Castroviejo \& Pascual, 1999), pero no se ha podido conocer el material que avala dicha cita. Aunque es cierto que el taxón existe en la flora cordobesa, es tan extraordinariamente raro que no había sido indicado en la Flora Vascular de Andalucía Occidental (Domínguez, 1987) ni ha sido recolectado recientemente. La cita, pues, confirma su presencia en el territorio.

Eryngium caespitiferum Font Quer \& Pau in Cavanillesia 4: 30 (1931)

CÓRDOBA. Poljé de La Nava, Cabra, 30SUG7851, 966 m.s.n.m., 29-VI-2018, J.A. Devesa \& J. López-Tirado (COFC 65780).

Especie descrita del norte de Marruecos ["In cacumine montis Lexhab (Gomara-Imp. Maroc.) ad $2100 \mathrm{~m}$ alt., solo argilloso-calcareo, ubi, floriferum, d. 21 julii 1930, legi"; Font Quer, 1931] y no recogida en la síntesis del género para Flora iberica (Nieto, 2003), pero cuya presencia en España fue dada a conocer por Triano (2010) en la provincia de Córdoba. Dicha población se localiza en el poljé de La Nava, en las proximidades de la localidad de Cabra, donde Triano (loc. cit.) la señaló como localmente abundante. Desafortunadamente, su fallecimiento prematuro (véase In Memoriam, Devesa, 2016) no ha permitido localizar material de este taxón, sin duda necesario para resolver en España la taxonomía del complejo de E. aquifolium Cav. Una nueva recolección en el mismo lugar, ha permitido constatar la notable abundancia de la especie en suelos hidromorfos, ya secos, junto a regatos y en los ribazos del nacimiento del arroyo Bailón, en compañía de Trifolium lappaceum L., Phleum nodosum L., Gastridium lainzii (Romero García) Romero Zarco, Festuca ampla Hackel, Aegilops neglecta Req. ex Bertol., entre otras especies. Esta población y la recientemente indicada por Cabezudo et al. (2016) para la Sierra de Camarolos (Málaga), son las únicas conocidas para el continente europeo.

Carum verticillatum (L.) W. D. J. Koch in Nova Acta Phys.-Med. Acad. Caes. Leop.-Carol. Nat. Cur. 12: 122 (1824)

Sison verticillatum L., Sp. PI.: 253 (1753)

CÓRDOBA. Arroyo del Risquillo, a $4 \mathrm{~km}$ de Belmez en la carretera a Hinojosa del Duque, 30SUH1041, 530 m.s.n.m., 26-V-2018, J.A.
Devesa \& G. Martínez-Sagarra (COFC 65781); ibídem, 17-VI-2018, J. A. Devesa (COFC 65788).

Especie del oeste de Europa y el norte de África, que aparece dispersa por la Península Ibérica (Gómez, 2003), y que en la provincia de Córdoba es muy rara, pues se conocía solo de las inmediaciones de Cardeña, en la comarca de Los Pedroches (Devesa \& Cabezudo, 1978). El nuevo hallazgo para las inmediaciones de Belmez constituye la segunda cita provincial del taxón, y extiende su área a Sierra Morena, más de $75 \mathrm{~km}$ al oeste-suroeste de la población conocida hasta la fecha.

\section{MONOCOTYLEDONEAE}

Schoenoplectus erectus (Poir.) Palla ex J. Raynal in Adansonia ser. 2, 16: 141 (1976) subsp. erectus

Scirpus erectus Poir. In Lam., Encycl. 6: 761 (1805) Scirpus uninodis (Delile) Coss. \& Durieu in Bory \& Durieu, Expl. Sci. Algérie 2: 310 (1868)

CÓRDOBA. Laguna de Los Arenales, término municipal de Puente Genil, 30SUG4944, 325 m.s.n.m., 29-VI-2018, J.A. Devesa \& J. LópezTirado (COFC 65785).

Especie de dudoso origen, tal vez naturalizada, que en la actualidad se encuentra distribuida por diversas regiones de África, SE de Asia, EE.UU., México, Argentina, Paraguay, y la Península Ibérica (Luceño \& Jiménez-Mejías, 2006). Aquí es rara, y se conoce -sub Scirpus uninodis- solo de Portugal (Luceño \& Jiménez-Mejías, loc. cit.) y de las provincias españolas de Jaén (Sánchez et al., 1997) y Huelva (Silvestre, 1980; Silvestre \& Cabezudo, 1980; Gallego, 1987; Sánchez Gullón \& Rubio, 2002; Valdés et al., 2006). La población cordobesa, muy nutrida, forma una amplia banda perilagunar en los suelos recientemente desecados, en los que la especie se acompaña de Marsilea strigosa Willd., Isoetes velatum A. Braun subsp. velatum, Damasonium polyspermum Coss., Lythrum borysthenicum (Schrank) Litv., y Polypogon maritimus Willd., entre otras. Su hallazgo en Córdoba no solo constituye la primera cita para la provincia sino que amplía el área en España de esta mal conocida especie.

Cenchrus longisetus M. C. Johnst. in Sida 1: 182 (1963)

Pennisetum villosum Fresen. in Mus. Senckenberg. 2: 134 (1837)

CÓRDOBA. Rabanales 21, entre el polígono y el Arroyo Rabanales, 30SUG4796, 114 m.s.n.m, 11-V-2018, J.A. Devesa (COFC 65779).

Neófito originario del noreste de África 
(Sánchez Gullón et al., 2010) que se ha naturalizado en diversas partes del mundo, entre ellas algunos países de Europa y el norte de África (vide Euro+Med PlantBase). En la Península Ibérica se conoce de Portugal (Amaral \& Rocha, 1998; Domingues de Almeida \& Freitas, 2006) $y$, sobre todo, de las áreas litorales del este de España (Bolòs \& Vigo, 2001; vide Anthos), también de las inmediaciones de El Portil, en la provincia de Huelva (Sánchez Gullón et al., 2010). Su hallazgo en las proximidades de Córdoba formando parte de herbazales nitrófilos, constituye la segunda cita para Andalucía occidental y la primera para la flora de la provincia de Córdoba.

Holcus mollis L., Syst. Nat. ed. 10, 2: 1305 (1759) CÓRDOBA. Río Yeguas, en su intersección con la carretera de Azuel a Fuencaliente, 30SUH8446, 528 m.s.n.m., 8-VII-2018, J.A. Devesa \& G. Martínez-Sagarra (COFC 65790).

Especie nativa en Europa y el noroeste de África (Romero Zarco, 1987), que en la Península Ibérica aparece abundante en la mitad septentrional, y mucho más dispersa en el sur (vide Anthos). Su presencia en la flora de la provincia de Córdoba es muy rara, pues se conocía hasta la fecha solo de las inmediaciones de Hornachuelos (Pinilla et al., 1998). La nueva cita extiende su presencia a la comarca de Pedroches, en el extremo nororiental del territorio, a más de $100 \mathrm{~km}$ de la única población conocida.

\section{Bibliografía}

Amaral Franco, J. Do \& Rocha Afonso, M. L. (1998). Nova Flora de Portugal III Gramineae, fasc. II (p. 233). Lisboa: Escolar Editora.

Anthos. Sistema de información de plantas de España [http://www.anthos.es/; consultado el día 10 de julio de 2018]

Bejarano, A., Gutiérrez, M. \& Vázquez, F. M. (2011). Aproximación al conocimiento de la flora neófita en la cuenca del Guadiana Internacional a su paso por Extremadura (España) Alentejo (Portugal). Folia Botanica Extremadurensis, 5, 5-27.

Bolòs, O. De \& Vigo, J. (2001). Flora dels Països Catalans, 4 (pp. 591-593. Barcelona: editorial Barcino.

Cabezudo, B., Casimiro Soriguer-Solanas, F., GarcíaSánchez, J. \& Pérez Latorre, A. V. (2016). Flora y vegetación de la zona de especial conservación (ZEC) de la Sierra de Camarolos (Málaga, España). Acta botanica malacitana 41: 163-246.

Cabezudo, B. \& Salvo, E. (1987). Marsilea. In, B. Valdés et al. (eds.) Flora Vascular de Andalucía Occidental 1 (p. 56). Barcelona: Ketres Editora S.A.

Castroviejo, S. \& Pascual, H. (1999). Lupinus. In, S. Talavera et al. (eds.) Flora iberica VII (pp. 251-260). Madrid: Departamento de Publicaciones del CSIC.
Crespo Villalba, M. B. \& Lledó Barrena, M.D. (1998). El género Limonium Mill. (Plumbaginaceae) en la Comunidad Valenciana: taxonomía y conservación. Colección Biodiversidad, $n^{\circ} 3$. Alicante: Ed. Generalitat Valenciana. Conselleria de Medi Ambient.

Delgado, A.J., Jiménez Sánchez, M. L. \& Medina, L. (2007). Notas pteridológicas. 52. Pilularia minuta Durieu, a new fern for Córdoba province (Andalusia, Spain). Acta botanica malacitana, 32: 247-248.

Devesa, J. A. \& Cabezudo, B. (1978). Contribución al estudio florístico del batolito de Los Pedroches (Córdoba). Lagascalia, 8: 53-103.

Devesa, J. A. (1987). Limonium. In, B. Valdés et al. (eds.) Flora Vascular de Andalucía Occidental, 1 (pp. 303307). Barcelona: Ketres Editora S.A.

Devesa, J. A. (2016). In Memoriam. Enrique Triano Muñoz (1967-2016). Acta botanica malacitana, 41: 343-347.

Domingues de Almeida, J. \& Freitas, H. (2006). Exotic naturalized flora of continental Portugal - A reassessment. Botanica Complutensis, 30: 117-130.

Domingues de Almeida, J. (2012). Flora exótica subespontânea de Potugal continental (Plantas Vasculares), ed. 5. Tesis. Coimbra.

Domínguez, E. (1987). Lupinus. In, B. Valdés et al. (eds.) Flora Vascular de Andalucía Occidental, 2 (pp. 177179). Barcelona: Ketres Editora S.A.

Erben, M. (2006). Limonium. In, S. Castroviejo et al. (eds.) Flora iberica, III (pp. 2-143). Madrid: Departamento de Publicaciones del CSIC.

Euro+Med PlantBase - the information resource for Euro-Mediterranean plant diversity [http://ww2.bgbm. org/EuroPlusMed/query.asp; consultado el día 10 de julio de 2018]

Fernández, P. (1986). Catálogo florístico de la cuenca hidrográfica del río Bembézar. Córdoba: Servicio de Publicaciones de la Universidad de Córdoba.

Font Quer, P. (1931). De flora occidentale adnotationes. Cavanillesia, 4: 25-31.

Gallego, M. J. (1987). Scirpus. In, B. Valdés et al. (eds.) Flora Vascular de Andalucía Occidental, 3 (pp. 229235). Barcelona: Ketres Editora S.A.

Gómez, D. (2003). Carum. In, G. Nieto et al. (eds.) Flora iberica, X (pp. 299-303). Madrid: Departamento de Publicaciones del CSIC.

González Castales, J. A. (2007). Plantas alóctonas invasoras en el Principado de Asturias. Consejería de Medio Ambiente, Ordenación del Territorio e Infraestructuras y Obra Social "La Caixa".

Herrera, M. \& Campos, J. A. (2010). Flora alóctona invasora en Bizkaia. Bilbao: Diputación foral de Bizkaia. Gráf. Munguía S.L.

Jiménez, M. \& E. Ruiz de Clavijo (1990). Nuevas áreas para la Flora de Andalucía Occidental. Lagascalia, 16: 132-145

López González, G. (1990). Rumex. In, S. Castroviejo et al. (eds.) Flora iberica, II (pp. 595-634). Madrid: Departamento de Publicaciones del CSIC.

López González, G. (2001). Los árboles y arbustos de la Península Ibérica e Islas Baleares, 2 vols. Madrid: Ed. Mundi-Prensa.

López Tirado, J., Tamajón, R. \& Hidalgo, P. J. (2016). 
Aportaciones a la flora vascular de la provincia de Córdoba, III (Andalucía, España). Flora Montiberica, 65: $48-56$

Luceño, M. \& Jiménez-Mejías, P. (2006). Notulae, taxinomicae, chorologicae, nomenclaturales, bibliographicae aut philologicae in opus "Flora Ibérica" intendentes, 7. Algunas novedades corológicas relevantes en ciperáceas ibéricas y baleáricas. Acta Bot. Malacitana, 31: 230-231.

Melendo Luque, M. (1998). De Mariani montis notualae, II. Lazaroa, 19: 173-178.

Moreno, J. C., Pataro, L. \& Pajarón, S. (2015). Atlas de los Pteridófitos de la Península Ibérica e Islas Baleares. Acta botanica malacitana, 40: 5-55.

Muñoz Álvarez, J. M., Vega, R. F., Delgado, J. M., Díaz, M. L. \& Alcántara, J. (2016). Contribución a la flora vascular de los humedales del sur de Córdoba (Andalucía, España). Flora Montiberica, 65: 8-16.

Nieto, G. (1987). El género Armeria (Plumbaginaceae) en la Península Ibérica: aclaraciones y novedades para una síntesis. Anales del Jardín Botánico de Madrid, 44: 319-348.

Nieto, G. (1990). Armeria. In, S. Castroviejo et al. (eds.) Flora iberica, II (pp. 642-721). Madrid: Departamento de Publicaciones del CSIC.

Nieto, G. (2003). Eryngium. In, G. Nieto et. al. (eds.) Flora iberica, X (pp. 36-60). Madrid: Departamento de Publicaciones del CSIC.

Nogueira, I. (1990). Phytolacca. In, S. Castroviejo et al. (eds.) Flora iberica, II (pp. 53-56). Madrid: Departamento de Publicaciones del CSIC.

Paiva, J. (1986). Marsilea. In, S. Castroviejo et al. (eds.) Flora iberica, I (pp. 66-69. Madrid: Departamento de Publicaciones del CSIC.

Pastor, J. (1987). Phytolacca. In, B. Valdés et al. (eds.) Flora Vascular de Andalucía Occidental, 1 (p. 166). Barcelona: Ketres Editora S.A.

Pinilla, R., Tamajón, R. \& Muñoz, J. M. (1998). Aportaciones a la flora de Córdoba. Acta botanica malacitana, 23: 260-269.

Prada, C. (1986). Isoetes. In, S. Castroviejo et al. (eds.) Flora iberica, I (pp. 15-20). Madrid: Departamento de Publicaciones del CSIC.

Prunier, F. \& J. M. Delgado (2010). Contribuciones a la flora vascular de Andalucía (España). 154. Marsilea strigosa Willd. En dos localidades de la provincia de Córdoba. Acta botanica malacitana, 35: 187-189.

Pujadas, A. (1986). Flora arvense y ruderal de la Provincia de Córdoba. Córdoba: Edit. Universidad de Córdoba, Escuela Técnica Superior de Ingenieros
Agrónomos.

Reyes, E., Martínez-Sagarra, G. \& Devesa, J. A. (2017). Novedades florísticas y corológicas para la provincia de Córdoba. Acta botanica malacitana, 42: 311-319.

Romero Zarco, C. (1987). Holcus. In, B. Valdés et al. (eds.) Flora Vascular de Andalucía Occidental, 3 (pp: 331-333). Barcelona: Ketres Editora S.A.

Royo Pla, F. (2006). Flora $i$ vegetació de les planes $i$ serres litorals compreses entre el riu Ebro i la serra d'Irta. Tesis Doctoral. Barcelona: Universitat de Barcelona, Facultat de Biologia..

Ruiz de Clavijo, E. \& Muñoz, J. (1985). Algunas plantas interesantes de la provincia de Córdoba. Acta botanica malacitana, 10: 79-83.

Sánchez Gullón, E., Fernández-Illescas, F., De las Heras, M. A., Jiménez Nieva, F. J., Rodríguez Rubio, P. \& Muñoz Rodríguez, A. F. (2010). Novedades florísticas para el litoral de Huelva y provincia. III. Lagascalia, 30: 461-472.

Sánchez Gullón, E. \& Rubio, J. C. (2002). Novedades florísticas para el litoral de Huelva y provincia. II. Lagascalia, 22: 7-19.

Sánchez Pascual, N., Melendo Luque, M., García Fuentes, A. \& Cano Carmona, E. (1997). Fragmenta chorologica occidentalia, 5938-5946. Anales del Jardín Botánico de Madrid, 55: 153.

Sanz, M., Dana, E. \& Sobrino, E. (2001). Aproximación al listado de plantas alóctonas invasoras reales y potenciales en España. Lazaroa, 22: 121-131.

Serra Laliga, L. (2007). Estudio crítico de la flora vascular de la provincia de Alicante: aspectos nomenclaturales, biogeográficos y de conservación. Ruizia, 19: 1-1414.

Silvestre, S. (1980). Números cormosómicos para la flora española. 150-154. Lagascalia, 9: 266-269.

Silvestre, S. \& Cabezudo, B. (1980). Notas Breves. 24. Scirpus uninodis (Delile) Boiss. Lagascalia, 9: 247248.

Triano, E. (2010). Nueva flora del Subbético cordobés. Córdoba: Publicación digital E. Triano (ISBN 978-84614-3417-6).

Trigo, M. M. (2000). Limonium. In, J. M. Sánchez de Lorenzo (coord.) Flora Ornametal Española, 2 (pp. 307-317). Sevilla: Ed. Junta de Andalucía, Ediciones Mundi-Prensa, y Asociación Española de Parques y Jardines Públicos.

Valdés, B., Carmona, I., Girón, V. \& Sánchez-Gullón, E. (2006). Notas taxonómicas y corológicas para la Flora de la Península Ibérica y El Magreb. Nota 119. Notas sobre la Flora de Doñana. 5. Nuevas áreas para el entorno de Doñana. Lagascalia, 26: 228-235. 